\title{
A Programme for Astrophysics
}

Theoretical Astrophysics :

Atomic Theory and the Analysis of Stellar Atmospheres and Envelopes. By S. Rosseland. (International Series of Monographs on Physics.) Pp. xix +355 . (Oxford : Clarendon Press ; London : Oxford University Press, 1936.) 25s. net.

THE author of this book is well known as the professor of astronomy in the University of Oslo. It is evident that the same broad outlook upon the future of his science that led him to found the Institute of Theoretical Astrophysics at Oslo, with its associated publication Astrophysica Norvegica, has inspired him also in planning this volume.

The first few chapters give a logically developed account of atomic theory, treated by wave mechanics approached from classical dynamics via statistical mechanics. It is taken as far as the theory of atomic multiplets, and followed by a short account of the quantum theory of radiation. So it provides in outline a necessary and sufficient basis in general physies for almost all the astrophysical theory which follows.

The central part of the book is naturally devoted to the theory of stellar atmospheres. Starting from the theory of propagation of radiation in an atmosphere, and the formation of the continuous spectrum, it passes on to the treatment of linewidths and total intensities. A special feature is the account taken from the outset of the possibility of cyclical atomic transitions between more than two quantum states. Prof. Rosseland was himself the first to realize their significance in his wellknown theory of bright lines in stellar spectra, and they have lately gained new importance in regard to the troublesome question of the central intensities of absorption lines. This theory is then linked on to that of thermal excitation and ionization in stellar atmospheres, which is specially associated with the names of Saha, Fowler, and Milne. By giving a simple unified rational interpretation of the whole sequence of stellar spectra, it probably still ranks as the most remarkable success in the application of atomic physics to astronomical observations. The effects of rotation, and of electric and magnetic fields, on the form of stellar lines are next considered. The occurrence of molecular bands in the spectra is also thoroughly discussed.

The last part of the book deals with the theory of stellar material lying outside the normal atmospheres. It proceeds from a consideration of the solar chromosphere and corona to the extended envelopes of some giant stars, then to gaseous nebulæ, and finally to the cosmic cloud. These all present new problems of mechanical and radiative equilibrium, amongst the latter being various intensely interesting questions connected with the occurrence of 'forbidden' lines.

The author's method in tackling any problem is first to describe clearly the general physical theory and the general mathematical methods that are required. In dealing with applications, he is, in this book, more concerned with key ideas, and with a careful statement of the assumptions and restrictions that have been made, than with details of results. This treatment will make the book particularly valuable to anyone beginning research in astrophysics. In the first place, it will put him in a position to refer intelligently to works of reference on physical subjects of the type of Fowler's "Statistical Mechanics" or the "Handbuch der Physik". In the second place, it will enable him to read the most recent work on the branches of theoretical astrophysics here treated, with a clear understanding of what has led up to it, and of the technique employed. Also the book will be welcomed by mathematical physicists wishing to gain an adequate idea of this field of application of their work.

The book has one characteristic which some readers may judge to be a deficiency. The astrophysical applications are in many cases left off at a seemingly unsatisfactory stage, and not completed by working out detailed theoretical results suitable for crucial observational tests. But this is apparently done by design. For, in spite of the almost startling successes of the subject, surveyed in the masterly introduction to the present volume, there is probably no part of it that will not repay fresh investigation using more powerful mathema. tical methods or more general physical models. The hope that this will be done, no less than that fresh problems will be attacked, is evidently behind the prefatory remark: "The aim of this work is to formulate a programme of theoretical astrophysics. . . A second volume is planned to give a similar view of the internal structure of the stars". Prof. Rosseland's attractive presentation of his programme should draw many recruits to the promising task of attempting it.

W. H. McC. 